\title{
Developing a vaccine against Zika
}

In this Editorial (BMJ 2016;355:i5923, doi:10.1136/bmj.i5923), the name of the third author was misspelt. The correct name is Vasee Moorthy. 